\title{
Extreme values in ionospheric radio propagation
}

Peter A. Bradley

Pandora, Farnham Common, Slough, Berks, U.K.

\begin{abstract}
Proposals are made for Earth-space and space-space paths to apply the term 'propagation degradation probability', analogous to 'basic circuit reliability' adopted within the Radiocommunication Sector of the International Telecommunication Union (ITU-R) to quantify the performance of HF terrestrial ionospheric radio circuits. The principal elements characterising propagation impairments are described and formulae and a table given by the ITU-R are reproduced relating many of these effects to the path total electron content. Expressions are provided enabling estimates of the probabilities of occurrence of user-specified extreme values to be estimated in terms of their median quantities and day-to-day variabilities. The need is stressed for additional data collection and analyses both to refine the median values and to derive the required reference variability factors.
\end{abstract}

Key words ionosphere - space radiowave propagation - circuit performance assessment

\section{Introduction}

Characterisation of extreme values for planning is important in many disciplines. For example, in insurance such figures are needed for risk assessment. In agriculture, they provide vital information about the yields of particular fields and the likely influence of application of specific fertilisers. In civil engineering, windloading factors are required when designing outdoor structures, and in oceanographic applications knowledge of largest waves likely to be encountered must be taken into account when designing offshore structures. Radio system design likewise needs information concerning extreme propagation effects that may arise.

Mailing address: Prof. Peter A. Bradley, Pandora, Scotlands Drive, Farnham Common, Slough, Berks SL2 3ES, U.K.
In all cases extreme values must be derived from past measurement data, either on the assumption that the future will be like the past, or with incorporation of a judicious trend factor. Sometimes the parameter being evaluated may be determined indirectly, using measurements of a different parameter, but the point to note is that always some form of measurement data is involved.

Measurement data sets, however large, have to be of finite size. Possibly for an infinitely large measurement data set the extreme value would be infinite (or zero), but that is not considered to be a useful result. More typically though, the largest (or smallest) values in successive data sets will not be the same, and so this raises the question how extreme values are to be determined. Traditionally expressions such as 'worst case' have been used, but this terminology is not considered here to be useful, and indeed is positively misleading. Worst case values would have to be successively revised using more and more data samples. Extreme values from within samples are known to be much less stable to characterise than say the median values. 
It is more precise then to express extreme values with regard to some selected probability of occurrence. For example, in deriving risks associated with artificial satellite failure, there may be a need to characterise the 'ten-year event'. For offshore structures the requirement may be to specify the 100 year wave. Of course the ten-year event or the 100 year wave may come tomorrow, but unless there is an a-priori reason to anticipate that situation, the statistical figure derived from past measurements remains the one to adopt.

From the foregoing it is seen that not only must measurement data be processed to yield extreme values, but also there must be a specification of what is meant by the word «extreme». That specification, which transposes to a particular probability of occurrence, has to be a subjective factor. It is derived with regard to the importance attached to the estimate, particularly where cost has to be balanced against risk.

One problem associated with extreme estimates is that often users of such information are unable to quantify the occurrence probabilities for which they want these to apply. Indeed, it is only meaningful to do so, if those people analysing the measurement data can specify these in a probabilistic fashion. Where that is not possible, which was often the case in the past, judicious 'safety factors' are introduced to allow for unforeseen factors. It is suggested here in the ionospheric propagation case that extreme values be determined for several different nominal probabilities of occurrence, such as $10 \%, 5 \%, 1 \%$ and $0.1 \%$.

Four separate propagation situations with potential ionospheric influences need to be addressed: i) reception of wanted signals from a ground-based transmitter, received at a grounded location after ionospheric reflection; ii) reception at a grounded location of co-channel and adjacent channel interfering signals from diverse sources; iii) reception at the ground or at a spacecraft of signals which traverse the ionosphere on an Earth-space link, and iv) reception at a spacecraft of signals originating from another spacecraft.

Except in the case of local interference not further addressed here, scenarios (i) and (ii) rely on the ionosphere for propagation support, whereas with (iii) and (iv) the presence of the ionosphere leads to signal corruption - change of ray path, delay, polarisation, attenuation etc. The treatment in these cases then needs to be different.

\section{HF reliability and compatibility}

ITU-R Recommendation P.842 (ITU-R, 2001a) defines the terms reliability and compatibility as the probabilities of satisfactory signal reception at $\mathrm{HF}$ in the presence of the natural and interference backgrounds, and when co-channel and adjacent channel signals are present respectively. For a particular radio circuit and in the presence of natural noise alone the reliability is known as Basic Circuit Reliability (BCR). When also interference is allowed for, the corresponding quantity is referred to as Circuit Reliability (CR). Formulae to compute these terms are given. BCR and CR are estimated in terms of predicted prevailing mean amplitudes and dayto-day variabilities of a wanted signal, and a background consisting of natural noise, manmade noise and interference, as appropriate. The required signal/background level must be specified. Compatibility is similarly determined from the mean amplitudes and variabilities of a wanted signal and a co-channel or adjacent channel unwanted signal. In this case, the protection ratio between wanted and unwanted signals must be chosen.

Table I reproduces the reference decile dayto-day deviations of signal strength from the monthly median values used in Recommendation P. 842. These values are combined on an rms basis with the corresponding withinthe-hour decile deviations of the signals and with the day-to-day and within-the-hour variations of the background, so that thereby the upper and lower decile deviations, $D_{u} S N$ and $D, S N$ respectively of the signal/ background ratio are given. For natural noise alone and with monthly median signal/noise ratio $S / N$, then, following Bradley and Bedford (1976), for a required signal/noise ratio $S / N$ and with all quantities expressed in decibels, 
Table I. Lower Decile (LD) and Upper Decile (UD) deviations from the predicted monthly median value of available receiver power of the wanted signal and interfering signals, arising from day-to-day variability.

\begin{tabular}{|c|c|c|c|c|}
\hline \multirow{2}{*}{$\begin{array}{l}\text { Geomagnetic latitude }\left(^{1}\right) \\
\text { Transmitting frequency/ } \\
\text { predicted basic MUF }\end{array}$} & \multicolumn{2}{|c|}{$<60^{\circ}$} & \multicolumn{2}{|c|}{$>60^{\circ}$} \\
\hline & LD & UD & LD & UD \\
\hline$\leq 0.8$ & 8 & 6 & 11 & 9 \\
\hline 1.0 & 12 & 8 & 16 & 11 \\
\hline 1.2 & 13 & 12 & 17 & 12 \\
\hline 1.4 & 10 & 13 & 13 & 13 \\
\hline 1.6 & 8 & 12 & 11 & 12 \\
\hline 1.8 & 8 & 9 & 11 & 9 \\
\hline 2.0 & 8 & 9 & 11 & 9 \\
\hline 3.0 & 7 & 8 & 9 & 8 \\
\hline 4.0 & 6 & 7 & 8 & 7 \\
\hline$>5.0$ & 5 & 7 & 7 & 7 \\
\hline
\end{tabular}

$\left({ }^{1}\right)$ If any point on that part of the great circle which passes through the transmitter and the receiver and which lies between control points located $1000 \mathrm{~km}$ from each end of the path, reaches a geomagnetic latitude of $60^{\circ}$ or more, the values of $>60^{\circ}$ have to be used.

the $\mathrm{BCR}$ as a percentage is given as:

$$
\begin{aligned}
B C R= & 130-80 /\left(1+\left(S / N-S / N_{r}\right) / D_{l} S N\right) \text { or } 100, \\
& \text { whichever is smaller } \\
& \text { for } S / N \geq S / N_{r} \\
= & 80 /\left(1+\left(S / N_{r}-S / N\right) / D_{u} S N\right)-30 \text { or } 0, \\
& \text { for } S / N<S / N_{r} \quad \text { whichever is greater }
\end{aligned}
$$

It is of note that for a particular transmitterreceiver path, separate reliability and compatibility values are derived because of changes in signal propagation and background levels for the different hours, months and solar epochs. So in choosing system parameters, e.g., transmitter power and types of antennas to achieve a desired service performance, there must also be subjective specification of what that desired service performance is. This is referred to as service probability. The service probability might for example be derived from just nighttime predictions over only a few months of a particular year, or it might embrace all hours over a full sunspot cycle, depending on the operational requirement. The above terms and the methods of their evaluation seem well established, and there would appear no need to seek to change these.

\section{PDP for Earth-space paths}

The determination of the signal components of BCR for a point-to-point $\mathrm{HF}$ radio circuit is a two-stage process. Firstly the probability of ionospheric signal support has to be evaluated using reference Maximum Usable Frequency (MUF) day-to-day variability statistics, and then given that propagation is possible, the probability has to be assessed that a required signal/noise ratio is met. In the case of Earth-space or space-space paths the situation is somewhat different in that it is supposed to a first order signals are received over line of sight trajectories with the various ionospheric effects representing a corruption of the free-space signals. The need is then to determine the probabilities that user specified tolerable propagation effect levels are met, rather than signal/noise values being achieved as at HF. 
Nonetheless, formulae to determine position within the associated probability distributions are the same in the two cases. So, instead of, but by analogy with BCR, here the term Propagation Degradation Probability (PDP) is judged more appropriate for space paths.

The principal ionospheric propagation effects on Earth-space paths may be listed as: Faraday rotation, propagation delay, refraction, absorption, dispersion and scintillation. For an effect e we may write, by analogy with eq. (2.1):

$$
\begin{aligned}
P D P_{\varepsilon}= & 130-80 /\left(1+\left(\varepsilon_{m}-\varepsilon_{r}\right) / D_{l} \varepsilon\right) \text { or } 100, \\
& \text { whichever is smaller } \\
& \text { for } \varepsilon_{m} \geq \varepsilon_{r} \\
= & 80 /\left(1+\left(\varepsilon_{r}-\varepsilon\right) / D_{u} \varepsilon\right)-30 \text { or } 0, \\
& \text { for } \varepsilon_{m}<\varepsilon_{r} \quad \text { whichever is greater }
\end{aligned}
$$

where $\varepsilon_{m}$ is the median value, $D_{l} \varepsilon$ and $D_{u} \varepsilon$ are reference lower and upper decile deviations from the median for effect $\varepsilon$ and $\varepsilon_{r}$ is the required value that must not be exceeded. In particular, with the reference decile deviations assumed given, eq. (3.1) requires knowledge of both the median and required values.

Recommendation P. 531 (ITU-R, 2001b) gives formulae and figures whereby median effect values may be estimated. Many such values are a function of the prevailing TEC. Alternative methods of determining TEC by electron-density height-profile integration are offered, using either the International Reference Ionosphere (IRI) produced by a COSPAR-URSI Task Group (Bilitza, 1990) or the model NeQuick (Leitinger et al., 1999; Hochegger et al., 2000), developed within COST251 (Leitinger and Feichter, 2000). The IRI model applies only for heights up to 2000 $\mathrm{km}$ whereas NeQuick, still under revision within COST271 (Radicella and Leitinger, 2001) and with its allied models COSTprof and NeUoG-plas (Leitinger, 1998a), now includes a plasmasphere topside (Leitinger, 1998b, 1999). Use of the IRI or NeQuick models enables median TEC to be given separately for ray paths in different geographical regions, and to show the dependence on time-of-day, season and epoch of the solar cycle, for the near full range of conditions for which they apply.

There are two independent approaches to TEC determination, the one involving profile integration as mentioned above, and the other from direct measurement of spacecraft signals. The latter method enables full allowances for electron content at the greater heights, but available data lack universal coverage. Various methods of combining the two data sets are being investigated within COST271 (Leitinger et al., 2001). Use of incoherent-scatter radar standardisation of GPS TEC-derived values is addressed in Lilensten and Cander (2001). There are obvious advantages in seeking to characterise TEC from a spacecraft using ionospheric tomography (Dabas and Kersley, 2001) or by means of occultation techniques (Garcia-Fernandes et al., 2001; Jakowski et al., 2001; Mitchell et al., 2001). It seems then likely that improved median TEC specifications will emerge in due course, in particular providing more realistic figures with greater spatial resolution than hitherto.

Some stated «estimated maximum» values of the principal ionospheric propagation effects likely to be encountered at $1 \mathrm{GHz}$ for a one-way ray path traversal of the ionosphere for an elevation angle of about $30^{\circ}$ are quoted in ITU-R Recommendation P.531 (ITU-R, 2001b) and these are reproduced in table II. For those parameters which exhibit a well-known frequency dependence and are directly related to TEC, values can readily be converted to corresponding figures for other elevation angles and frequencies.

It is suggested here though that instead one should follow the eq. (3.1) statistical approach and endeavour to model the decile deviations of the various effects. As a first step this involves trying to specify the TEC decile deviations. Moreover, it seems probable that these decile TEC would better come from limited direct measurements, rather than from profile integration, because in the profile case, although the variabilities of the separate 'anchor values' such as layer peak densities and heights are generally well known, the correlations in the changes among them are not established. 
Table II. ITU-R Recommendation P.531 'estimated maximum values'.

\begin{tabular}{llc}
\hline \multicolumn{1}{c}{ Effect } & \multicolumn{1}{c}{ Magnitude } & Frequency dependence \\
\hline Faraday rotation & $108^{\circ}$ & $1 / f^{2}$ \\
Propagation delay & $0.25 \mu \mathrm{s}$ & $1 / f^{2}$ \\
Refraction & $<0.17 \mathrm{mrad}$ & $1 / f^{2}$ \\
Variation in direction of arrival & $0.2 \mathrm{~min}$ of arc & $1 / f^{2}$ \\
Absorption (polar cap absorption) & $0.04 \mathrm{~dB}$ & $\sim 1 / f^{2}$ \\
Absorption (mid-latitude) & $<0.01 \mathrm{~dB}$ & $1 / f^{2}$ \\
Dispersion & $0-4 \mathrm{~ns} / \mathrm{MHz}$ & $1 / f^{3}$ \\
Scintillation & Limited amplitude information only quoted & \\
\hline
\end{tabular}

\section{PDP for space-space paths}

Ionospheric effects may be estimated from ray-tracing results through an appropriate ionospheric model such as NeUoG-plas to derive the corresponding TEC values on satellite-tosatellite paths (Hochegger and Leitinger, 2000). At this time it is not clear how median TEC may be characterised, but some amalgamation of separate results to give representative figures will no doubt be necessary. Despite variability between cases, it ought to be possible to provide results whereas none are currently available. Likewise, the study of TEC variability on spacespace paths remains to be carried out.

\section{Combining PDP values for different propagation effects}

Signals received over an HF point-to-point ionospheric path experience time spread and frequency dispersion as well as attenuation due to passage through the ionosphere. Hence, particularly in the case of digital transmissions, circuit performance criteria have to take account not only of the probability of achieving a user-specified required signal/ background ratio, but also specified time spreads and frequency dispersions that must not be exceeded. Bradley and Muhtarov (2000), first proposed via ITU-R (1997), have developed formulae to combine the BCR values for each of these effects on the assumption that they are independent of oneanother. For effects $\varepsilon_{1}, \varepsilon_{2}$ and $\varepsilon_{3}$ we have that the combined BCR is

$$
B C R=B C R_{\varepsilon 1} \cdot B C R_{\varepsilon 2} \cdot B C R_{\varepsilon 3} .
$$

Adaptation of this formulation may also be applied to evaluations for Earth-space and spacespace paths when considering independent effects such as absorption and scintillation, leading to

$$
P D P=P D P_{\varepsilon 1} \cdot P D P_{\varepsilon 2} \cdot P D P_{\varepsilon 3}
$$

but in the case of effects directly dependent on TEC, these have to be regarded as completely correlated, in which case the PDP is taken as the smallest of those separately evaluated for the different effects.

\section{Combining PDP values for different times and paths}

PDP may be determined as wanted. PDP estimates may be used either to predict the performance of an established circuit, or to permit the design specification to meet stated performance objectives. In either case, PDP values are likely to need combining for different times and different satellite locations. This means that criteria to be achieved may well involve multiple effect tolerances for stated fractions of days, hours, months and years, as well as for a certain percentage of satellite positions. An example of a performance specification might be that the group delay must not exceed $0.2 \mu$ s for more than $5 \%$ of the nights 
between March 2003 and September 2005 for a polarorbiting spacecraft over $90 \%$ of the world ocean area

The formulae given above permit these types of figures to be derived. On the other hand, it can be appreciated that for some applications, having regard also to uncertainties in ionospheric predictions, a single all-embracing effect estimate achieved say for $95 \%$ of occasions and covering all times and satellite locations might be favoured. This is a matter of user choice.

\section{Conclusions}

Extreme value estimates in ionospheric radio propagation should be determined from variability statistics. The concept of Basic Circuit Reliability (BCR) developed to characterise the probability that the signal/noise ratio for an HF circuit attains a required threshold is extended to Earth-space and space-space paths giving the Propagation Degradation Probabilities (PDP) that acceptable levels are achieved of the different propagation effects related to Total Electron Content (TEC) such as refraction, dispersion and Faraday rotation, as well as arising from ionospheric absorption and scintillation. Formulae are quoted giving PDP when multiple tolerance criteria must be met, both for uncorrelated and fully correlated effects. The particular need is identified to permit these evaluations for the derivation of reference decile deviations of TEC from the monthly median values, and it is concluded that such information will best come from analyses of direct measurement data, rather than from electrondensity height profile integration.

\section{REFERENCES}

BILITZA, D. (1990): The International Reference Ionosphere 1990, National Space Science Data Center Report 90-22, Greenbelt, Maryland.

BRADLEY, P.A. and C. BEDFORD (1976): Prediction of HF circuit availability, Electron. Lett., 12, 32-33.

BRADLEY, P.A. and P. MUHTAROV (2000): Basic circuit reliability for digital HF ionospheric communications, Fisica de la Tierra, 12, 353-361.

DABAS, R.S. and L. KERSLEY (2001): Radio tomographic imaging as an aid to modelling of ionospheric electron density, in Proceedings of the COST271 Sopron
Workshop (CD-ROM obtainable from the project Secretariat at: angela.vernon@rl.ac.uk).

GARCIA-FERNANDES, M., M. HERNANDES-PAJARES, J.M. JUAN, J. SANZ and R. ORUS (2001): Ionospheric data combination as a way to improve electron density estimates, in Proceedings of the COST271 Sopron Workshop (CD-ROM obtainable from the project Secretariat at: angela.vernon@rl.ac.uk).

HOCHEGGER, G. and R. LEITINGER (2000): Inversions of satellite to satellite electron content: simulation studies with NeUoGplas, Phys. Chem. Earth, 25 (4), 353-357.

HoCHEGGER, G., B. NAVA, S. RADICELlA and R. LEITINGER (2000): A family of ionospheric models for different uses, Phys. Chem. Earth, 25 (4), 307-310.

ITU-R (1997): Basic circuit reliability for digital modulation systems, Doc. 3L/33, Rapporteur Group 3L-RG1, International Telecommunication Union, Geneva.

ITU-R (2001a): Computation of reliability and compatibility of HF radio systems, ITU-R Recommendation P.842-2, International Telecommunication Union, Geneva.

ITU-R (2001b): Ionospheric propagation data and prediction methods required for the design of satellite services and systems, ITU-R Recommendation P.531-6, International Telecommunication Union, Geneva.

JAKOWSKI, N., A. WEHRENPFENNIG, S. HEISE, CH. REIGBER and H. LUHR (2001): GPS radio occultation measurements of the ionosphere on CHAMP - initial results, in Proceedings of the COST271 Sopron Workshop (CD-ROM obtainable from the project Secretariat at: angela.vernon@rl.ac.uk).

LEITINGER, R. (1998a): NeUoG-plas - an easy to use global electron density model for the ionosphere and plasmasphere, in Proceedings of the 2nd Workshop of COST251, 140-150.

LEITINGER, R. (1998b): A magnetic field aligned approach to model the topside $F_{2}$ layer, Adv. Space Res., 22, 789-792.

LEITINGER, R. (1999): Magnetic field aligned modeling for the upper $F$ region and for the plasmasphere, Phys. Chem. Earth, 24, 299-303.

LEITINGER, R. and E. FEICHTER (2000): COSTTEC - the electron content monthly median map adopted by COST251, Phys. Chem. Earth, 25 (4), 311-314.

LEITINGER, R., S. RADICELLA, B. NAVA, G. HOCHEGGER and J. HAFNER (1999): NeQuick-COSTprof-NeUoG-plas, a family of 3D electron density models, in Proceedings of the 4th Workshop of COST251, 75-89.

LEITINGER, R., S. RADICELLA and B. NAVA (2001): Electron density models for assessment studies - new developments, in Proceedings of the COST271 Sopron Workshop (CDROM obtainable from the project Secretariat at: angela.vernon@rl.ac.uk).

LILENSTEN, J. and LJ. CANDER (2001): Calibration of the TEC derived from GPS or from models using the EISCAT radar, in Proceedings of the COST271 Sopron Workshop (CDROM obtainable from the project Secretariat at: angela.vernon@rl.ac.uk).

MitCHELL, C.N., C. CHONG and P.S. SPENCER (2001): Observing electron concentration using radio-occultation and groundbased GPS observations, paper presented at the COST271 Workshop, Sopron, Hungary, 25-29 September 2001.

RADICELLA, S.M. and R. LEITINGER (2001): The evolution of the DGR approach to model electron density profiles, $A d v$. Space Res., 27 (1), 35-40. 